
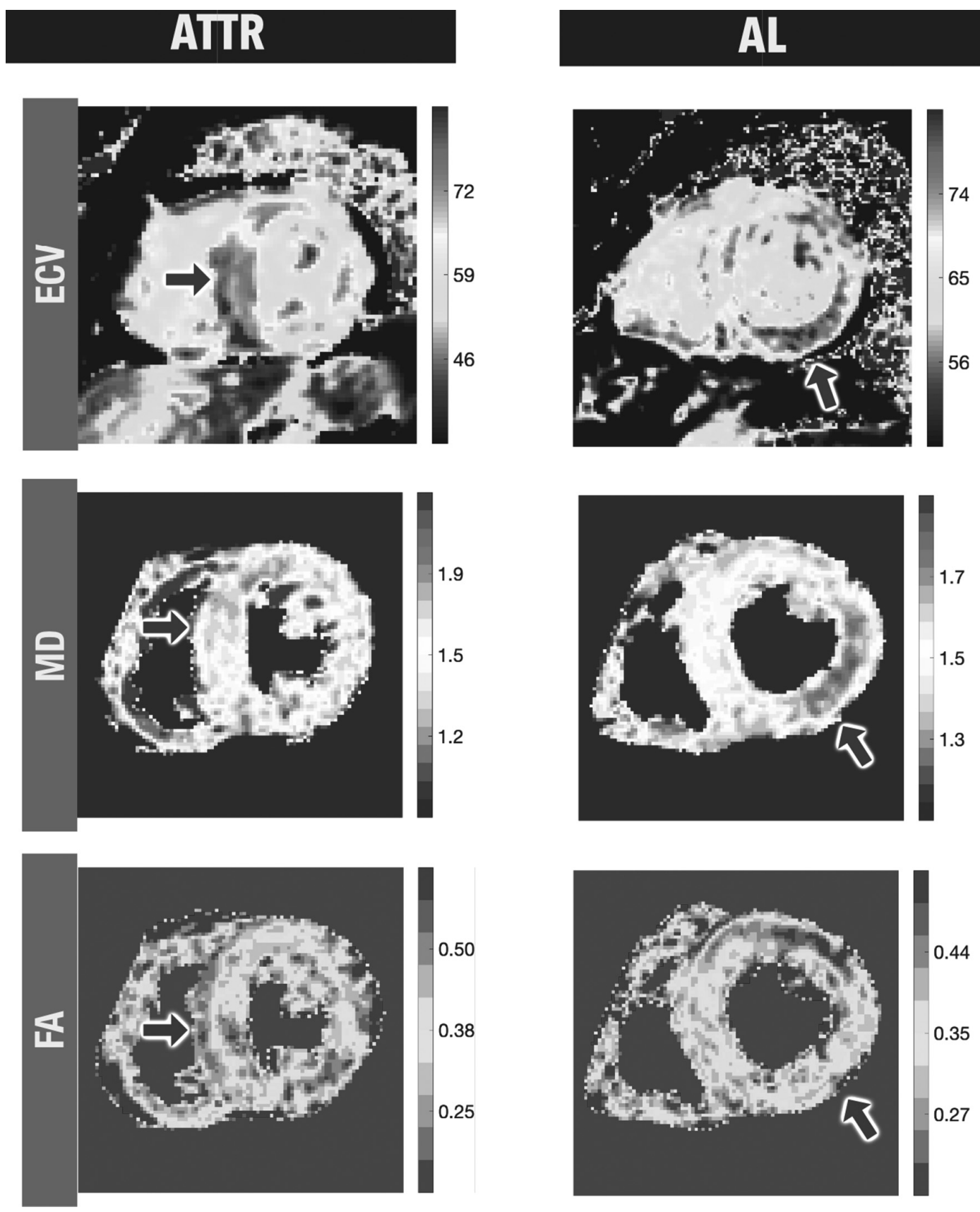

Abstract 7 Figure $2 \mathrm{MD}$ and FA map the location and extent of amyloid deposition

Example maps from an AL and an ATTR patient are shown. In the top row ECV maps reflect the burden of amyloid. MD maps in the second row show areas of abnormally elevated MD in orange and red. Abnormally reduced FA is orange-green (third row). The MD and FA maps show colocation of the areas of elevated MD and reduced FA with amyloid as shown by ECV.

\section{A PROSPECTIVE LONGITUDINAL FOLLOW UP STUDY USING T1 AND T2 MAPPING SEQUENCES AND TWELVE- SEGMENT MYOCARDIAL ASSESSMENT TO IDENTIFY AND MONITOR MYOCARDIAL INFLAMMATION IN MYOSITIS}

${ }^{1} \mathrm{D}$ Bromage, ${ }^{1} \mathrm{~A}$ Nabeebaccus, ${ }^{1} \mathrm{~K} \mathrm{O}^{\prime}$ gallagher, ${ }^{2} \mathrm{~K} \mathrm{Le},{ }^{2} \mathrm{~L}$ Pearce, ${ }^{2} \mathrm{~A}$ Millin, ${ }^{3} \mathrm{P}$ Kellman, ${ }^{1} \mathrm{P}$ Gordon, ${ }^{1} \mathrm{D}$ Sado. ${ }^{1}$ Kings College London, London, UK; ${ }^{2}$ Kings College Hospital, London, UK; ${ }^{3}$ National Institute of Health (Home), Washington, USA

\subsection{6/heartinl-2019-BSCMR.8}

Background Myositis is a systemic autoimmune condition causing skeletal muscle inflammation and fibrosis as well as extra skeletal involvement. Cardiac involvement is well described and has morbidity and mortality. It can be diffuse or focal which can make assessment using conventional imaging techniques more difficult. To date the incidence is poorly defined using conventional cardiac investigations.

Purpose To investigate the reproducibility of a 12 segment ventricular myocardial model of $\mathrm{T} 1$ and $\mathrm{T} 2$ mapping in healthy volunteers and then to use this methodology in patients with myositis, comparing it to the current non invasive gold standard of troponin I level for cardiac involvement and then monitor how it changes with time and treatment. Methods 19 Healthy volunteers were scanned at 1.5T. T1 (using a MOLLI sequence) and T2 mapping were performed 
in basal and mid short axis. The images were analysed by blinded readers to assess the reproducibility of the 12 segment myocardial segmentation approach.

Patients were recruited from the Rheumatology department which is a regional centre for myositis. Patients with cardiac symptoms or elevated troponin I were recruited. Recruits underwent CMR with tissue mapping and late gadolinium enhancement at the first visit with 2 subsequent non-contrast mapping studies followed by a final gadolinium enhanced CMR at 12 months.

All patient scans were performed on the same 1.5T scanner and analysed by the same reader. In order to mitigate for bias a second reader, blinded to the clinical details including the troponin, repeated the analysis on $50 \%$ of studies. The T1 and T2 were calculated for 12 segments in each scan.

Results Reproducibility of a 12 segment model for analysing tissue mapping sequences is excellent with a coefficient of variability of $1.4 \%$ for T1\% and $2.6 \%$ for T2. Myositis patients with elevated troponin I had a significantly higher mean T1 and T2 than healthy volunteers $(\mathrm{p}<0.0001$ both). Myositis patients with elevated troponin $\mathrm{i}$ have a significantly higher T1 and T2 than myositis patients with normal troponin I $(\mathrm{p}<0.01$ both). Patients with myositis and a negative troponin I have a significantly higher $\mathrm{T} 1$ and $\mathrm{T} 2$ than healthy volunteers (T1 $\mathrm{p}<0.01, \mathrm{~T} 2 \mathrm{p}<0.05)$. There is a significant reduction in T1 and T2 values in patients with treatment over 8 months $(\mathrm{p}<0.05$ both).

Conclusions A twelve segment model for myocardial T1 and T2 assessment is highly reproducible which is important in this disease process as there can be either focal or diffuse myocardial involvement. T1 and T2 are strongly associated with troponin I positivity with both likely to be a reflection of active inflammation in this disease process and improve with immunesuppression therapy fairly quickly.

\section{TRAIN THE AI LIKE A HUMAN OBSERVER: DEEP LEARNING WITH VISUALISATION AND GUIDANCE ON ATTENTION IN CARDIAC T1 MAPPING}

Qiang Zhang, Konrad Werys, Elena Lukaschuk, Iulia Popescu, Evan Hann, Stefan Neubauer, Vanessa M Ferreira, Stefan K Piechnik. OCMR, University of Oxford Centre for Clinical Magnetic Resonance Research

\subsection{6/heartjnl-2019-BSCMR.9}

Background Artificial intelligence (AI) is increasingly used in diagnostic imaging. Deep convolutional neural networks (CNN) are able to learn from datasets presented to them, and then provide independent interpretations on new cases, but often without traceability of how they came to the conclusions. Such 'black box' behaviour is not desirable for clinical use. We propose the concepts of visualising and guiding the AI attention in an application to artefact detection in cardiac T1-mapping - a critical quality assurance step for clinicallyrobust T1 determinations.

Method We utilise the emerging AI attention visualisation. This serves as an 'eye tracker' and reveals where the neural network 'looks' when scoring artefacts in T1 mapping, and adds an essential accountability aspect to the CNN by producing additional evidence to validate the decision making process. Beyond simply observing the perception, we developed a technique to provide direct guidance on the attention of the $\mathrm{CNN}$, by telling the machine which region to look at, very similar to training a human observer.

Results We demonstrate an application in automated T1 mapping artefact detection of the 6 AHA segments in mid-ventricular slices (figure 1a). The AI 'eye tracker' detected an illtrained CNN paying attention to features not desired for the assigned tasks (figure 1b). A well-trained CNN learned from the training data to pay attention to the corresponding

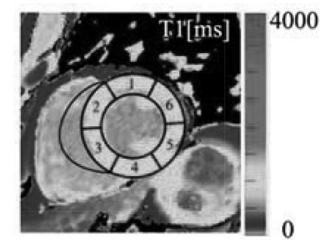

(a) AHA segmental model

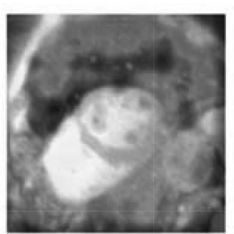

(b) Attention of an ill-trained CNN
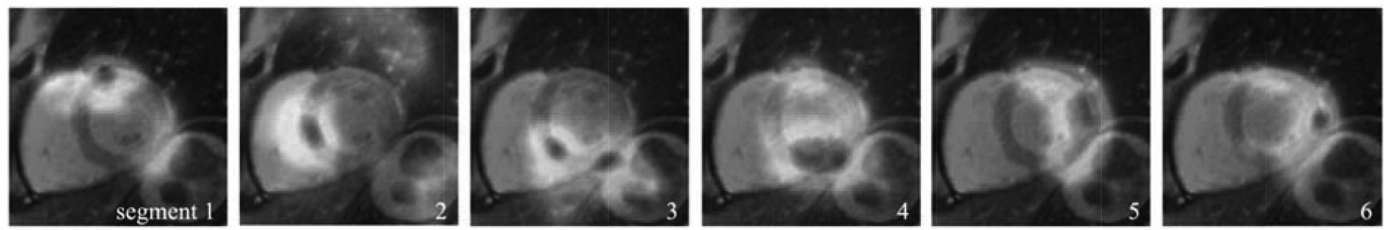

(c) Attention of a well-trained $\mathrm{CNN}$ when detecting artefacts in 6 segments
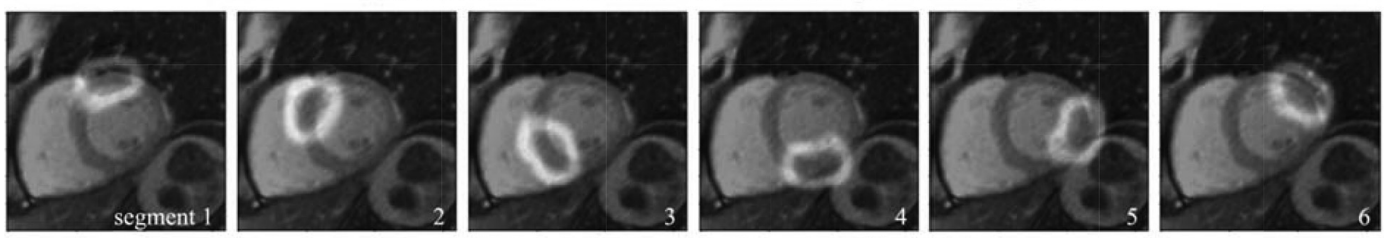

(d) $\mathrm{CNN}$ trained with proposed attention guidence technique

Abstract 9 Figure 1 Attention visualisation and guidance in detecting T1 mapping artefacts in the 6 AHA segments (a), which reveals that (b) an ill-trained CNN looked at the features irrelevant to the tasks, (c) a well-trained CNN highlighted the segments but with distraction by other image features, and (d) with attention guidance in training the CNN highlighted the segments more accurately 